\title{
Efficient Clustering Technique for Cooperative Wireless Sensor Network
}

\author{
Kaushlendra Kumar Pandey \\ Jaypee University of Information Technology,Solan \\ Email: kaushlendre@gmail.com \\ Neetesh Purohit \\ Indian Institute of Information Technology,Allahabad \\ Email: neetesh.purohit@gmail.com \\ Ajay Agarwal \\ Jaypee University of InformationTechnology,Solan \\ Email: ajay.jop@gmail.com
}

\begin{abstract}
The nodes of a wireless sensor network (WSN) collect the data of interest from the deployment area and cooperatively send it to sink in multiple hops. Recently, virtual multiple input multiple output (V-MIMO) technique has been introduced in which the node cooperation is extended to form a virtual antenna array and communication is accomplished using space time block coding. The present work extends the scope of $\mathrm{V}$ MIMO by proposing a new clustering technique. It creates the temporary cluster heads and their respective cooperative nodes, collects information from them and then the final clustering is accordingly done. The simulation result shows significant improvement in the energy efficiency of the WSN.
\end{abstract}

Index Terms - Cooperative Communication, Virtual MIMO, Wireless Sensor Network, Energy Efficiency.

\section{INTRODUCTION}

The nodes of WSN possess a sensing unit, a computing unit and a wireless transceiver. It needs special energy efficient protocols for collecting data from the field, aggregating information and transferring it to the target device, known as sink or the base station. The end user, located at a distant place, can access the data from the base station. Once deployed, the WSN need to work for maximum time in the unattended environment. Thus, the issue of energy conservation (thus life time extension) is regarded as the primary objective of WSN design. Low life time is the biggest deterrence of WSN technology which underlines the need of further improvement for faster adaption of WSN technology. However, it has already been recognized as a promising technology for the applications where manual deployment of sensor units, laying down wires from these units to a central place and the maintenance of the network is very difficult or impractical. The habitat monitoring, agriculture, space exploration, military systems, industrial automation etc. are some of the potential fields for WSN deployment [12]. The clustering operation enhances the energy efficiency of WSN by several folds thus it has always been a crucial issue in WSN research [3]. Several efficient clustering techniques exists for standard WSN configuration (single input single output (SISO) WSN) in which the transceivers have single antenna and each transmission is independently accomplished.

In V-MIMO WSN's the transceivers still possesses single antenna but two or more nodes cooperate to accomplish the transmission using space time block codes. Thus, the clustering techniques developed for SISO WSN's are not optimal in V-MIMO WSN's. A clustering technique for V-MIMO WSN's is presented in this work. It involves two steps, first the cluster heads are identified using any SISO clustering technique e.g. Low Energy Adaptive Clustering Hierarchy (LEACH), then a potential cooperative node is discovered for each cluster head. In second step the clustering is done in an efficient manner. Several proposals exists for realizing V-MIMO systems [4] but the integration of clustering technique with $\mathrm{V}$ MIMO WSN is first time presented in this work.

This paper is organized as follows. In the section II we have discussed the related works that have been done in this area. The rules for the node cooperation and cooperative node selection, possible ambiguities and ways of handling them are presented in section III. The simulation framework is presented in section IV which is followed by results and discussion in section $\mathrm{V}$. The conclusion is presented in section VI.

\section{RELATED WORKS}

Clustering is the one of the important method to conserve the energy in sensor network. Every cluster has a leader which is also known as cluster head. Various clustering technique have been introduced by the researchers for ad-hoc network [5-9]. Some other clustering technique has been proposed for wireless 
sensor network [10-14]. These clustering technique also depend upon the node deployment methodology and also the network constrains. The cluster head is selected in a pre-determined manner decided by the network designer. Clustering also has advantages in term of scalability a clustering algorithm also helps to route data from one cluster to another and it leads to reduce the routing table stores in a particular node [15]. Clustering technique also helps to reduce the bandwidth because clustering reduces the redundant data transfer in a network [16]. This technique also helps in the network management methodology which leads to improve the battery life time of sensor nodes. A cluster head also helps to reduce the data processing and eliminating the redundant data in a cluster and performs the data aggregation technique [17].

MIMO technique has been adopted in cellular and other leading wireless networks for getting better data rate but V-MIMO is more suitable for WSN's [18]. Multiple antennas should not be hosted in sensor node transceiver for keeping the physical dimensions of the node as small as possible. Instead, a cooperative communication mechanism should be explored to develop a V-MIMO WSN [19] [20] so that benefits of MIMO becomes available which should be reaped in the form of reduced transmit power thus enhanced life time of WSN. The conventional three node model was proposed in [21].

Cooperative diversity was introduced by Wornell et. al. [18] for wireless network. The cooperative diversity was introduced for both technique that is decode and forward and first amplify the signal and then forward. The other name of cooperative diversity is user cooperative diversity [22]. In network nodes share their antenna in such way that they make virtual antenna array [23] so in the network when node transmit the data most of the node present in the network are over here this information and then they again send this information to the base station. A. Blestsasey et Al [24] introduced a new concept of cooperation. In this technique network comprise of multiple relay and node choose the appropriate relay for the cooperation in the available number of relays. Aitor del Coso, considered a multi-hop WSN with sensors grouped in cooperative clusters in which the routing is performed by layer-3 hierarchical protocols [25]. Xin Zhang H. Vincent Poor and Mung propose [26] a virtual MIMO based technique to implement in the sensor network to transfer message from few sensor node to the base station. They have considered that sensor motes are disseminated in network and each sensor node has different power constrained and the sense data by the nodes is also different. The sensor node jointly used their power to send data to base station. In the other approach used in [27] a combination of cooperative MIMO with data aggregation is used to reduce energy degradation in network, whole process of communication was separated into two parts, first part is called as local communication and another is called as long haul communication. Zhong Zhou proposed another approach [27] this technique is divided in two phases: Intra cluster Broadcasting and Inter cluster Cooperative Transmission. In Sudharman K
Jayaweera's [28] work, there are two sets of node one is data gathering node and other is data collection node, data collection node send the sense data to the data gathering node using any channel access technique for example time division multiple access scheme.

It has been observed that MIMO implemented WSN's is more energy efficient than SISO, if we are destined to provide better quality of service to the end user it affects energy efficiency of WSN. There is inherent trade-off between better quality of service and life time of wireless sensor network [29]. Cooperative node selection is an important part in MIMO-WSN; it is specific problem of game theory where $\mathrm{CN}$ node selection is based on the mutual profit of the cluster head and cooperative node selection. Non cooperative game theory is also used in MIMO-WSN [30] [31]. In clustered network a node in a particular cluster jointly cooperates with each other to reduce the energy consumption they also intended to reduce their own energy consumption. In the game theory which studies the complex relation of mutual friendliness and selfishness between the players [32], Comprehensive surveys on the application of coalition formation in games theory have been done in [33]. There is only little work available the coalition formation of game theory in WSN [33] and [34]. If focused on enhancing the life time of WSN for a specific quality of service [33].

\section{THE PRoposed Clustering TECHNIQUe}

The proposed clustering involves following two phases.

(a) Potential Cluster head selection (using LEACH) and cooperative node identification

(i). After random deployment, the LEACH protocol should run for cluster head selection [35] which gives the potential cluster heads. (Some of these nodes will actually become the cluster head whereas others need to give-up their status based on the result obtained from next phase thus these are designated as potential cluster heads.) Instead of declaring themselves as cluster head (as happened in LEACH) they should start to search the best neighbor for establishing the cooperation by broadcasting the invitation message to join them.

(ii). The nodes which receive the invitation message should stop the probability counter of becoming cluster head, collect the information of its neighbor and send it to the closest (in case of multiple invitations) cluster head.

(iii). Nodes will select their respective cluster head based on the received signal strength (RSSI).

(iv). The cluster head compare its neighbor list with received neighbor list from each neighbor. The node having minimum number of common neighbors should be selected as the cooperative node for forming the virtual antenna array.

(v). Nodes having the minimum number of common neighbors will more suitable to become the cooperative node because of their less correlated data. 
(b) The cluster formation

(i). The potential cluster head will now broadcast its clusterhead status alongwith list of all nodes which should join it. The list has special status to the node which has been chosen to become cooperative node.

(ii). The cooperative node also declare itself to be the cluster head with a list of uncommon nodes which can be easily obtained by comparing the list of its neighbors and the neighbor list received from the primary cluster head. These neighbors of should now treat the cooperative node as an independent cluster head. Ecah member node should stop the probability counter of becoming cluster head and send its consent of joining the cluster.

(iii). A member node may have got this message from more than one node. It should give the consent to the nearest clusterhead and ignore the other messages.

The proposed scheme has following features:

a) The average cluster size obtained from this technique should be approximately double than that obtained from LEACH.

b) The average distance from member node to the cluster head should remain approximately same in both cases.

c) Space time coding ensures low average power consumption of this bigger cluster as the datarate enhancement is not needed.

d) Better data aggregation is possible and the overall transmitted data per node is reduced which also saves the transmission energy.

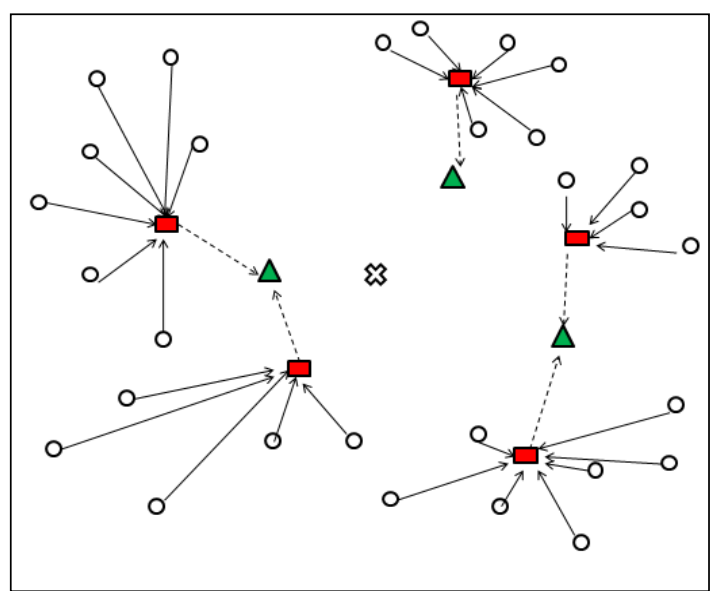

Fig. 1 an example snapshot of the final clusters after the random deployment

Figure 1 shows a snapshot of the final clusters formed by above method. The green nodes (may be triangular) are $\mathrm{CH}$, red (may be square ) are the cooperative nodes and white (circular) are the member nodes. The members are shown to be connected by arrows to the CH's where as dotted arrows are used to represent the $\mathrm{CH}-\mathrm{CN}$ link.

Various possible ambiguities associated with above scheme and their solutions are discussed here under. a) If a node is isolated in the network and does not receives any link from any node present in the network. In such a case, node will wait for next round so that there is a good possiblity to find a nearby cluster head in the network.

b) After LEACH if no node is free to link with the cooperative nodes then the member node should leave the membership of previous cluster or clusterhead and join the cooperative node if it is closer otherwise simply ignore the request.

c) The cooperative nodes should not become the cluster-head in the next few rounds. In LEACH, when a node become cluster head in the previous round, then it makes its $\mathrm{C}_{\mathrm{i}}(\mathrm{t})=0$,i.e., it cannot become the cluster head. Same rule should be used for the cooperative nodes.

\section{THE PROBLEM FORMULATION}

The whole problem can be divided in to two parts one is per node energy consumption after clustering and second is per node energy consumption during clustering and summation of above will provide the total energy consumption in per node in the network and other calculation regarding the network parameter like number of rounds to which proposed protocol will run.

We have assumed that uniform clustering have been taken in the network and after the second phase a cluster head will only comprise the cooperative node now $n_{1}$ is the number of cooperative node in cluster and $n$ is number of node attached to each cooperative node (obviously $n>n_{1}$ ) than number of node in the cluster is $\left(\mathrm{nn}_{1}+1\right)$ now each node in a cluster sensed $\mathrm{K}$ number of bits in given time period it is already been explained that in a cluster nodes are closely spaced there is high possibility of correlation among the sensed data by the node so we have introduced level of data compression in network first data compression will take place at the cooperative node level and second compression will be done by the cluster head level, two type of communication will take place after the clustering in the network, first is local communication or intra cluster communication the other will called as long haul communication in which the cluster head will send data to base station with the help along with the cooperative node. For the local communication we have assumed a square path loss model with additive white Gaussian noise and for the long haul communication we have assumed Rayleigh fading channel we have taken space time block code for long haul cooperative communication. Total energy degradation can be separated into two parts:

$$
E_{\text {total }}=E_{\text {local }}+E_{\text {longhaul }}
$$

\section{A) Energy Degradation in Local Communication}

Thus $\mathrm{n} * \mathrm{n}_{1}$ nodes transmit there data to there $\mathrm{n}_{1}$ cooperative node and it is being assumed that data transfer rate is $R_{\text {intra }}$ and if the bandwidth is $B$ and the 
constellation size is $\mathrm{c}$ than transmission data rate will equal to

$$
R_{\text {intra }}=c^{*} B
$$

The total energy consumption is distinguished into two parts first is energy consumption in the power amplifier circuitry and other is energy consumption in other circuitry thus energy consumption in sending data to the cooperative node by the nodes:

$$
E_{1}=\frac{n * n_{1} * P_{S I S O} * K}{R_{\text {intra }}}+\frac{n * n_{1}\left(P_{t}+P_{r}\right) * K}{R_{\text {intra }}}
$$

We have calculated the link budget $P_{\text {siso }}$ on the basis of relationship that has provided in [35] and [36].

$$
P_{\text {siso } 1}=(1+\alpha) E_{\text {intra }} R_{\text {intra }} \frac{(4 \pi)^{2} d_{1}^{2}}{G_{t} G_{r} \lambda^{2}} M_{l} N_{f}
$$

In the above equation $\alpha=\left(\frac{\zeta}{\eta}\right)-1$ where $\zeta$ is magnitude of peak to average ratio $\eta$ specified the draining efficiency of power amplifier it has been assumed that all nodes associated with cooperative node are having almost equal separation with cooperative node to simplify this problem it is also been assumed that each node are equally spaced with cooperative node if the transmitter antenna gain is equal to $G_{t}$ and receiver antenna gain is a $\mathrm{G}_{\mathrm{r}}$ the hardware is another significant factor that we have to conceive for estimating overall energy consumption $\mathrm{M}_{\mathrm{l}}$ represents the same link margin and $\mathrm{N}_{\mathrm{f}}$ is equal to noise level of receiver circuitry

$$
N_{f}=\frac{N_{r}}{N_{0}}
$$

$N_{r}$ Represents power spectral density of total noise at the receiver and $\mathrm{N}_{0}$ is power spectral density of thermal noise at $27{ }^{\circ} \mathrm{C}$ (Room Temperature). Peak to average ratio $\zeta$ is concerned with constellation size of the modulation scheme that has been adopted. In proposed protocol it is being adopted the MQAM (multiquadrature amplitude modulation) for intra cluster communication or local communication [36]

$$
\zeta=3\left(\frac{2^{b / 2}-1}{2^{b / 2+1}}\right)
$$

To compute value of $P_{\text {siso }}$ that is the total power depleted in the power amplifier we also have to calculate the value of $E_{\text {intra }}$ that is per bit energy that necessitated for given bit error rate for multiquadrature amplitude modulation (MQAM) the average value of bit error rate in MQAM modulation when the constellation size $(b)=2$

$$
\beta \approx Q\left(\sqrt{ }\left(2 \Gamma_{\text {intra }}\right)\right.
$$

$Q$ is, $\mathrm{Q}$ function which is expressed in terms of mathematical equation as:

$$
Q(x)=\frac{1}{\sqrt{(2 \pi)}} \int_{x}^{\infty} e^{-\frac{t^{2}}{2}} d t
$$

$\Gamma_{\text {intra }}$ Denotes the value of instantaneous SNR and can be written as:

$$
\Gamma_{\text {intra }}=\frac{E_{\text {intra }}}{N_{0}}
$$

Putting the value of equation 9 into equation 7 it will find the value of $E_{\text {intra }}$ for given bit error rate.

This is the energy consumption when sense data is being transmitted from sensor node to cooperative node now we will have to calculate the energy consumption that take place from the cooperative node to the cluster head for this aim we use same equation and assumption that we have taken to calculate the consumption from node to the cooperative node let the energy consumption is $E_{2}$ :

$$
\begin{gathered}
E_{2}=\frac{n 1 * K * P_{\text {siso } 2}}{R_{\text {intra }}}+\frac{n 1 *\left(P_{t}+P_{r}\right) * K}{R_{\text {intra }}} \\
P_{\text {siso } 2}=(1+\alpha) \frac{E_{\text {intra } 2} * R_{\text {intra }} *(4 \pi)^{2} * d 2^{2}}{G_{t} * G_{r} * \lambda^{2}} * M_{2} N_{f 2}
\end{gathered}
$$

Now to estimate energy consumption in data aggregation for this purpose it is being consider the grid based data aggregation technique for data aggregation and this will be equal to:

$$
E_{\text {com }}=n K E_{\text {comp } 1}+n_{1} K E_{\text {comp } 2}
$$

After the overall data aggregation at the cluster head the net amount of data that have to send to the base station will be K. Now the cluster head will broadcast this $\mathrm{K}$ bits to the cooperative node to get the spatial diversity thus energy consumption for broadcasting phase will be equal to the:

$$
E_{\text {bro }}=P_{\text {siso }} \frac{K}{R_{\text {intra }}}+\left(P_{t}+n_{1} P_{r}\right) \frac{K}{R_{\text {intra }}}
$$

Thus net energy consumption in the inter cluster communication will equal to the:

$$
E_{\text {local }}=E_{1}+E_{2}+E_{\text {bro }}+E_{\text {comp }}
$$

\section{B) Energy Consumption in Long haul communication}

The method to calculate the long haul energy consumption is being adopted [38]

In the long haul communication cluster head along with the cooperative node will send data to base station, data will send with the help of space time block code to base station the total energy consumption in long haul communication is provide by following equation:

$$
E_{l h}=P_{M I M O} \frac{K}{R_{l h}}+\left(\left(n_{1}+1\right) P_{t}+P_{r}\right) \frac{K}{R_{l h}}
$$


Where $P_{\text {MIMO }}$, is power consumed in power amplifier at transmitter, $\mathrm{R}_{\mathrm{lh}}$ is representing the transmission bit rate defined as $\mathrm{R}_{\mathrm{lh}}=\mathrm{R}_{\mathrm{s}} * \mathrm{~B}$ where encoding strategy is done at spatial rate of $R_{s}$. In current scenario value of $R_{s}=1 / 2$, because we are using orthogonal space time block code for data transmission. In this protocol it is being assumed that base station is located at centre of network thus number of clusters form in the network are almost equally spaced from the base station, distance of each cluster from base station is D, and it is being considered that it is almost equal distance from the each cooperative node because in a cooperative nodes have almost equal distance from the base station thus power consumption by power amplifier is given by equation:

$$
P_{M I M O}=(1+\alpha) E_{l h} R_{l h} \frac{\left(4 \pi^{2}\right) D^{2}}{G_{t} G_{r} \lambda^{2}} M_{l} N_{f}
$$

Where $E_{l h}$ is the average energy required per bit for provided bit error rate in order to estimate bit error rate in MIMO system, when MQAM is used for MQAM the value of $b=2$

$$
\begin{gathered}
\beta_{l h}=\mu\left[Q\left(\sqrt{ }\left(2 \Gamma_{l h}\right)\right]\right. \\
=\int_{0}^{\infty} Q\left(\sqrt{ }\left(2 \Gamma_{l h}\right) f\left(\Gamma_{l h}\right) d_{\Gamma l h}\right.
\end{gathered}
$$

Where $\mu_{\mathrm{h}}(\mathrm{x})$ represents expected value of $\mathrm{x}$ over given channel vector $h$ and $\Gamma_{\mathrm{lh}}$ is the value of instantaneous magnitude of signal to noise ratio for cooperative MIMO system considered in [4] which is given by the equation:

$$
\Gamma_{l h}=\frac{E_{l h}}{(n 1+1) N_{0}}\|h\|^{2}
$$

In this equations $\left(n_{1}+1\right)$ node are transmitting data to the base station and the channel vector can be represents as:

$$
h=\left[h_{1} h_{2} \ldots \ldots . h_{n+1}\right]
$$

Every channel gain is only depends upon its Rayleigh fading. In the long haul communication $\left(\mathrm{n}_{1}+1\right)$ node are transmitting data to terminus station according to space time block code.

Here it is being taken the upper bound for demanded energy per bit from the Chernoff bound [39] and that is equal to

$$
\mathrm{E}_{\mathrm{lh}} \leq \frac{(\mathrm{n} 1+1) \mathrm{N}_{0}}{\beta_{\mathrm{lh}}^{1 /\left(\mathrm{n}_{1}+1\right)}}
$$

We have estimated overall energy consumption in intra cluster communication and energy degrade in long haul communication thus the overall energy consumption $E_{\text {total }}$ can be computed with assistance of equation(14) and equation (15):

$$
E_{\text {total }}=E_{\text {local }}+E_{l h}
$$

From the all above equations and solving the overall energy consumption:

$$
\begin{array}{r}
E_{\text {total }}=\frac{n n_{1}}{R_{\text {intra }}}\left[K P_{\text {SISO } 1}+\left(P_{t}+P_{r}\right) K\right]+\frac{n_{1}}{R_{\text {intra }}}\left[K P_{\text {siso } 2}+\left(P_{t}+\right.\right. \\
\left.\left.P_{r}\right) K\right]+(n+n 1) K E_{\text {comp }}+\frac{P_{\text {siso }} K}{R_{\text {intra }}}+\frac{\left(P_{t}+n 1 P_{r}\right) K}{R_{\text {intra }}}+\frac{P_{M I M O} K}{R_{\text {lh }}} \\
+\left[\left(n_{1}+1\right) P_{t}+P_{r}\right] \frac{K}{R_{\text {lh }}}
\end{array}
$$

This is over all energy consumption in cluster but when we calculate the energy consumption per node we have to divide the number of nodes in the different part of the equation because different number of node are participating in different form of energy transfer from cluster to terminus station.

$$
\begin{gathered}
E_{\text {node }}=\frac{n}{R_{\text {intra }}}\left[K P_{\text {SISO } 1}+\left(P_{t}+P_{r}\right) K+\frac{1}{R_{\text {intra }}}\left[K P_{\text {siso } 2}+\left(P_{t}+\right.\right.\right. \\
\left.\left.P_{r}\right) K\right]+\left[\frac{P_{\text {Siso }} K}{R_{\text {intra }}}+\frac{\left(P_{t}+n 1 P_{r}\right) K}{R_{\text {intra }}}\right] \frac{1}{(n 1+1)}+\frac{P_{M I M O} K}{R_{\text {lh }}(n 1+1)}+[(n 1+ \\
\left.1) P_{t}+P_{r}\right] \frac{K}{R_{\text {lh }}(n 1+1)}+K E_{\text {comp }}
\end{gathered}
$$

\section{The Result AND Discussion}

In the figure given below it is shown that when we deal the per node energy consumption from cooperative node to the cluster head $\left(d_{2}\right)$ and in case of no cooperation from node to the cluster head per node energy consumption is reduced in comparison to the no cooperation, and in the following figure we have shown that per node energy consumption and number of node join the cooperative node and in case of no cooperation number of node join the cluster head.

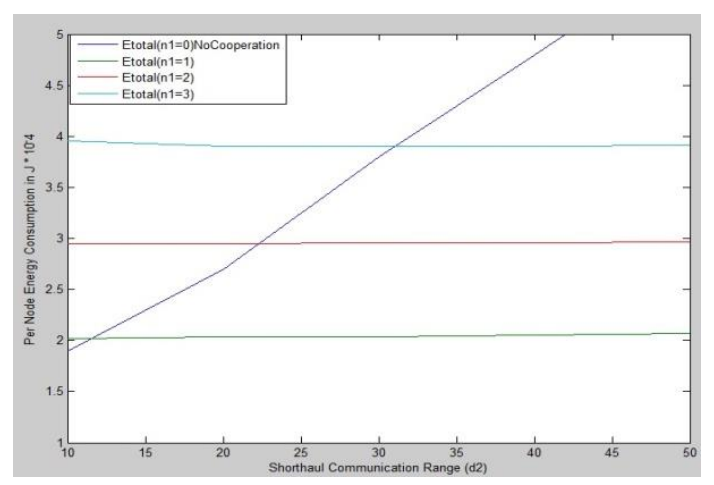

Fig. 2

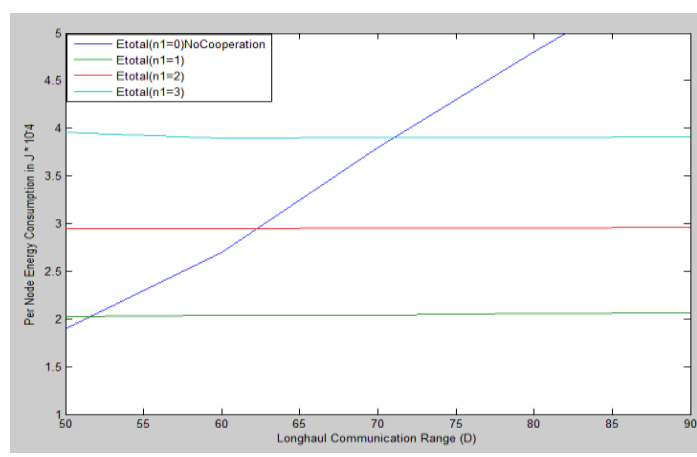

Fig 3 
The above figure shows the overall per node energy consumption versus long haul communication range and it represents that as the long haul communication range increases per node energy consumption reduces drastically.

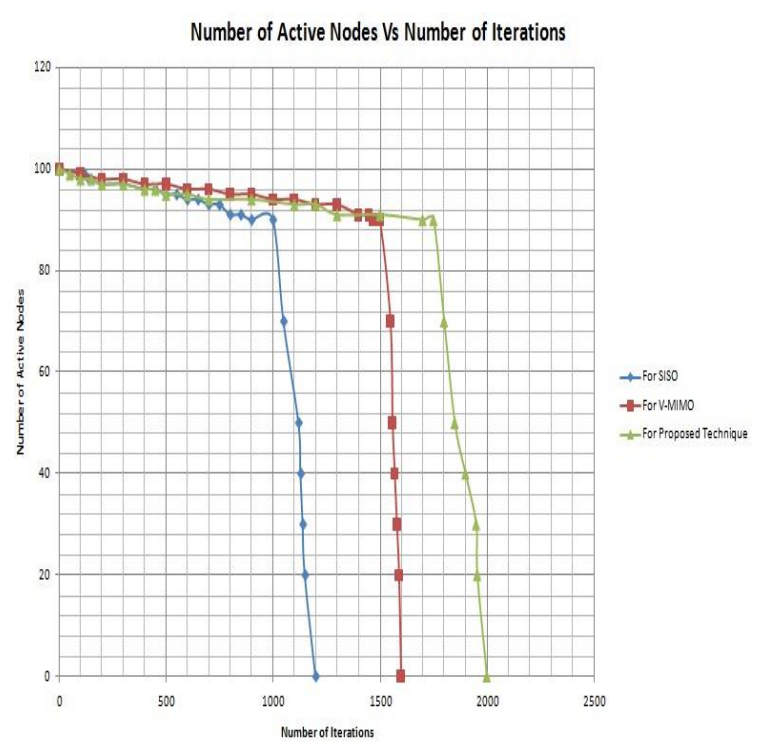

Fig: 4. Number of active nodes Vs. Number of iterations

Figure 2 shows that initial performance, till 1000 iterations, of 3 schemes are more or less similar and SISO system can work till 1000 iterations. V-MIMO based WSN shows an improvement and it can function fairly well up to 1500 iterations. When proposed scheme is incorporated with V-MIMO then its life is enhanced by 300 iterations.

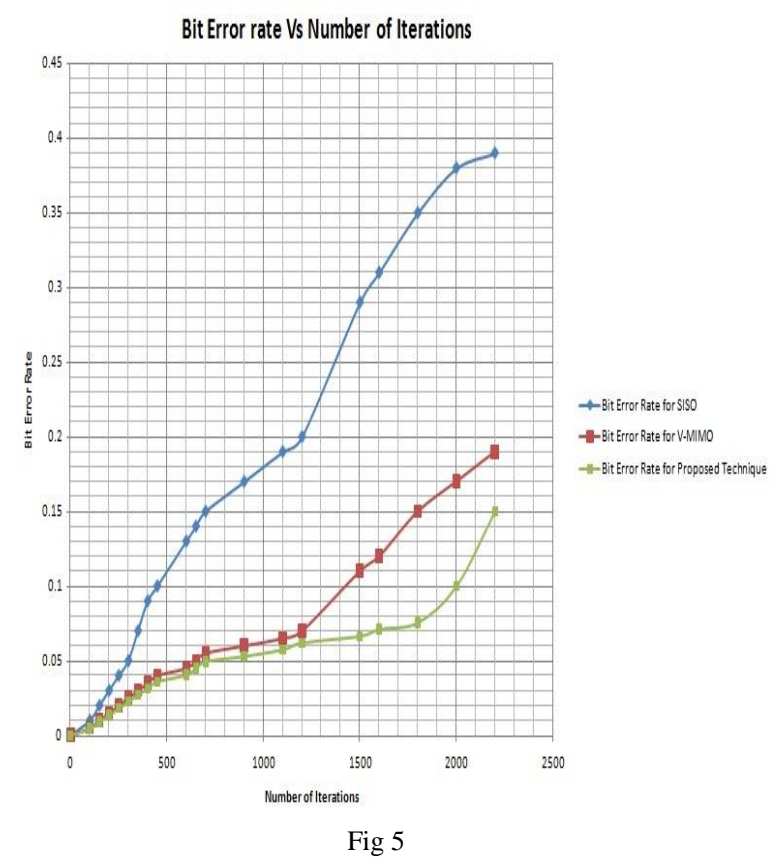

Figure 3 shows the BER which is measured during operation phase after clustering. Up to 1200 iterations the V-MIMO and the proposed techniques show similar performance but after that an exponential enhancement is observed in V-MIMO but the proposed technique shows fairly low BER up to1700 iterations.

\section{CONCLUSION AND FUTURE WORK}

The features of V-MIMO based WSN are exploited in this work for developing a new clustering technique. Its performance has been found better than conventional hierarchical clustering protocol. It also outperforms the conventional single input single output systems. Development of efficient MAC protocol, development of efficient sleep-wake protocol etc. for V-MIMO based WSN may be future extensions of the present work. Better algorithm for information fusion may be developed for clustering and operational phase (after clustering) then the precision and performance of WSN will definitely improve. It is out of the scope of the present work.

\section{REFERENCES}

[1] I. Akyildiz, W. Su, Y. Sankarasubramaniam, and E. Cayirci, "A survey on sensor networks," IEEE Commun. Mag., vol. 40, no. 8, pp. 102-114, Aug. 2002.

[2] Ankit K. Jain, Ankit Khare, and Kaushlendra K. Pandey. Developing an efficient framework for real time monitoring of forest fire using wireless sensor network. In Proc. Of the 2nd IEEE Int'l Conf. on Parallel, Distributed and Grid Computing (PDGC-2012), 2012. ISBN 978-14673-2922-4.

[3] Eleni Klaoudatou, Elisavet Konstantinou, Georgios Kambourakis, and Stefanos Gritzalis,- A Survey on Cluster-Based Group Key Agreement Protocols for WSNs, IEEE COMMUNICATIONS SURVEYS \& TUTORIALS, VOL. 13, NO. 3, THIRD QUARTER 2011.

[4] S. Cui, A. J. Goldsmith, and A. Bahai, "Energy efficiency of MIMO and cooperative MIMO techniques in sensor networks," IEEE J. Sel. Areas Commun., vol. 22, no. 6 , pp. 1089-1098, Aug. 2004.

[5] V. Kawadia, P.R. Kumar, Power control and clustering in Ad Hoc networks, in: Proceedings of IEEE INFOCOM, San Francisco, CA, March 2003.

[6] M. Chatterjee, S.K. Das, D. Turgut, WCA: a Weighted Clustering Algorithm for mobile Ad Hoc networks, Cluster Computing 5 (2) (2002) 193-204.

[7] A.D. Amis, R. Prakash, T.H.P. Vuong, D.T. Huynh, MaxMin Dcluster formation in wireless Ad Hoc networks, in: Proceedings of IEEE INFOCOM, March 2000.

[8] A.B. McDonald, T. Znati, A mobility based framework for adaptive clustering in wireless ad-hoc networks, IEEE Journal on Selected Areas in Communications 17 (8) (1999) 1466-1487.

[9] S. Basagni, Distributed clustering algorithm for ad-hoc networks, in: Proceedings of the International Symposium on Parallel Architectures, Algorithms, and Networks (ISPAN), Fremantle, Australia, June 1999.

[10] G. Gupta, M. Younis, Load-balanced clustering in wireless sensor networks, in: Proceedings of the International Conference on Communication (ICC 2003), Anchorage, Alaska, May 2003.

[11] S. Bandyopadhyay, E. Coyle, An energy efficient hierarchical clustering algorithm for wireless sensor networks, in: Proceedings of the 22nd Annual Joint Conference of the IEEE Computer and Communications Societies (INFOCOM 2003), San Francisco, California, April 2003. 
[12] S. Ghiasi, A. Srivastava, X. Yang, M. Sarrafzadeh, Optimal energy aware clustering in sensor networks, Sensors Magazine MDPI 1 (1) (2004) 258-269.

[13] O. Younis, S. Fahmy, HEED: A Hybrid, Energy-Efficient, Distributed clustering approach for Ad Hoc sensor networks, IEEE Transactions on Mobile Computing 3 (4) (2004) 366-379.

[14] W.B. Heinzelman, A.P. Chandrakasan, H. Balakrishnan, Application specific protocol architecture for wireless microsensor networks, IEEE Transactions on Wireless Networking (2002).

[15] K. Akkaya, M. Younis, A survey on routing protocols for wireless sensor networks, Elsevier Journal of Ad Hoc Networks 3 (3) (2005) 325-349.

[16] Y.T. Hou, Y. Shi, H.D. Sherali, On energy provisioning and relay node placement for wireless sensor networks, IEEE Transactions on Wireless Communications 4 (5) (2005) 2579-2590.

[17] K. Dasgupta, K. Kalpakis, P. Namjoshi, An efficient clustering-based heuristic for data gathering and aggregation in sensor networks, in: Proceedings of the IEEE Wireless Communications and Networking Conference (WCNC, 2003), New Orleans, LA, March 2003.

[18] J. N. Laneman, D. N. C. Tse, and G. W. Wornell," Cooperative Diversity in Wireless Networks: Efficient Protocols and Outage Behavior,"IEEE Transactions on Information Theory, vol. 50, no. 12, pp. 3062-3080, Dec. 2004.

[19] S. M. Alamouti, -A simple transmit diversity technique for wireless communications," IEEE Journal on selected areas in communications, vol. 16, no. 8, pp. 1451-1458, Oct. 1998.

[20] KK Pandey, A Jain, and R Dekar, “The Energy Efficient Cooperative MIMO Technique for Hierarchical Clustering Protocol", International Journal of Computer Applications 57(9):23-28, November 2012.

[21] A. Nosratinia, T. E. Hunter, and A. Hedayat, -Cooperative Communication in Wireless Networks," IEEE Communications Magazine, Oct. 2004.

[22] A. Sendonaris, E. Erkip, and B. Aazhang, -User cooperation diversity-part 1: system description, || IEEE Trans. Commun., vol. 51, no. 11, pp. 1927-1938, Nov. 2003.

[23] M. Dohler, E. Lefranc, and H. Aghvami, - Space-time block codes for virtual antenna arrays, \|PIMRC, Lisbon, Portugal, Sep. 2002.

[24] A. Bletsas, A. Khisti, D. P. Reed, and A. Lippman, -A Simple Cooperative Diversity Method Based on Network Path Selection," IEEE Journal on Selected Areas in Communications, vol. 24, no. 3, pp. 659-672, Mar. 2006.

[25] Aitor del Cos Umberto Spagnolini and Christian Ibars, Cooperative Distributed MIMO Channels in Wireless Sensor Networks IEEE JOURNAL ON SELECTED AREAS IN COMMUNICATIONS, VOL. 25, NO. 2, FEBRUARY 2007.

[26] Xin Zhang, H. Vincent Poor and Mung Chiang Optimal Power Allocation for Distributed Detection Over MIMO Channels in Wireless Sensor Networks IEEE TRANSACTIONS ON SIGNAL PROCESSING, VOL. 56, NO. 9, SEPTEMBER 2008.

[27] Z. Zhou,S. Zhou, S. Cui, and J. Cui, "Energy-efficient cooperative communication in a clustered wireless sensor network," IEEE Trans. Veh. Technol., vol. 57, no. 6, pp. 3618-3628, Nov. 2008.
[28] S. K. Jayaweera, -Virtual MIMO-based cooperative communication for energy-constrained wireless sensor networks," IEEE Transaction on Wireless Communications, vol. 5, no. 5, pp. 984-989, May 2006.

[29] H. Nama, M. Chiang, and N. Mandayam, "Utility-lifetime trade-off in self-regulating wireless sensor networks: a cross-layer design approach," in Proc. 2006 IEEE ICC.

[30] S. Sengupta, M. Chatterjee, and K. A. Kwiat, "A game theoretic framework for power control in wireless sensor networks," IEEE Trans. Comput., vol. 59, no. 2, pp. 231242, 2010.

[31] H. Ren and M. Q.-H. Meng, "Game-theoretic modeling of joint topology control and power scheduling for wireless heterogeneous sensor networks," IEEE Trans. Automation Science Eng., vol. 6, no. 4, pp. 610-625, 2009.

[32] W. Saad, Z. Han, M. Debbah, A. Hjprungnes, and T. $\mathrm{Ba}$,sar, "Coalitional game theory for communication networks," IEEE Signal Process. Mag. vol. 26, no. 5, pp. 77-97, 2009.

[33] D. Wu, Y. Cai, and J. Wang, "A coalition formation framework for transmission scheme selection in wireless sensor networks," IEEE Trans. Veh. Technol., vol. 60, no. 6, pp. 2620-2630, 2011.

[34] O. N. Gharehshiran and V. Krishnamurthy, "Coalition formation for bearings-only localization in sensor networks-a cooperative game approach," IEEE Trans. Signal Process., vol. 58, no. 8, pp. 4322-4338, 2010.

[35] W. B. Heinzelman, A. P. Chandrakasan, and HariBalakrishnan, -An Application-Specific Protocol Architecture for Wireless Microsensor Networks," IEEE Trans. Wireless Commun., vol. 1, no. 4, Oct. 2002.

[36] J. G. Proakis, Digital Communications, 4th ed. New York: McGraw-Hill, 2000.

[37] S. Cui, A. J. Goldsmith, and A. Bahai, "Modulation optimization under energy constraints," in Proc. IEEE Int. Conf. Commun., Anchorage, AK, May 2003, pp. 28052811.

[38] Qiang Gao, Yi Zuo, Jun Zhang, and Xiao-Hong Peng "Improving Energy Efficiency in a Wireless Sensor Network by Combining Cooperative MIMO With Data Aggregation" IEEE TRANSACTIONS ON VEHICULAR TECHNOLOGY, VOL. 59, NO. 8, OCTOBER 2010.

[39] H. Chernoff, "A measure of asymptotic efficiency for tests of a hypothesis based on the sum of observations," Ann. Math. Statist., vol. 23, no. 4, pp. 493-507, Dec. 1952.

\section{Authors' Profiles}

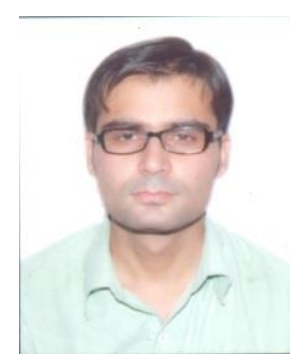

Kaushlendra Kumar Pandey received B-Tech degree from Gautam Budh Technical University Lucknow (Uttar Pradesh, India) in Electronics and Communication in 2010 and M-Tech from Indian Institute of Information Technology Allahabad (Uttar Pradesh, India) in speciation of wireless communication and computing in 2012.

$\mathrm{He}$ is currently working as Assistant Professor in the Department of Electronics and Communication in Jaypee University of Information Technology Solan (Himanchal Pradesh) India. 


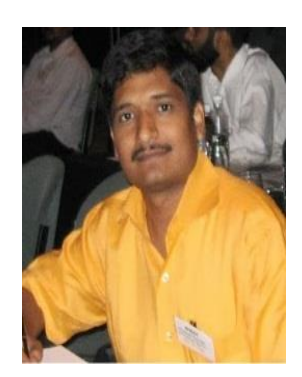

Dr Neetesh Purohit is working as Assistant Professor in Indian Institute of Information Technology Allahabad (Uttar Pradesh,India).

His area of interest is modern wireless communication system development and deployment of wireless sensors network and antenna engineering.

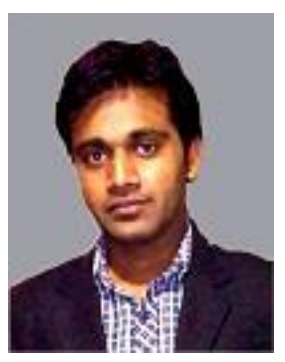

Ajay K. Agrawal receieved his B-Tech from Gautam Budh Technical University Lucknow (Uttar Pradesh, India) in Electronics and Communication in 2011. He received the M.Tech degree (with honors) in optoelectronics and optical communication from Indian Institute of Technology, New Delhi, India, in 2013. He joined Jaypee University of Information Technology, Waknaghat, H.P., India, in 2013, where he is currently working as an Assistant Professor in the Electronics and Communication Department.

How to cite this paper: Kaushlendra Kumar Pandey, Neetesh Purohit, Ajay Agarwal,"Efficient Clustering Technique for Cooperative Wireless Sensor Network", IJCNIS, vol.6, no.10, pp.40-47, 2014. DOI: 10.5815/ijcnis.2014.10.05 